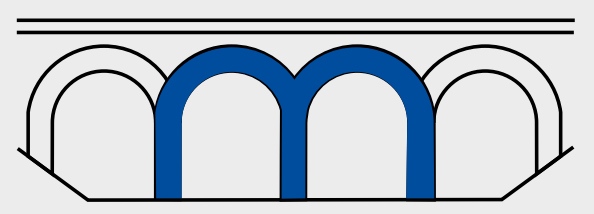

Berichte des Instituts für Massivbau

Ralf Herrmann

Simulation und Regelung

von Resonanzversuchsständen

zur Untersuchung

der Bauteilermüdung

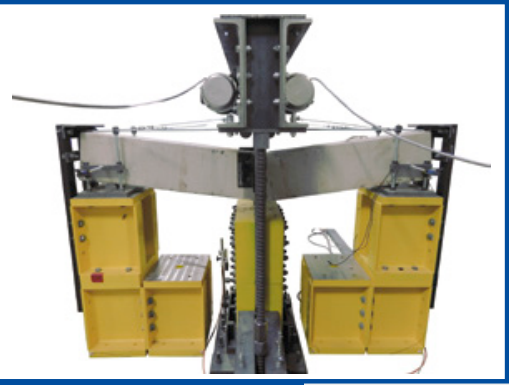

Fraunhofer IRB_Verlag 


\section{Berichte}

des Instituts für Massivbau der Leibniz Universität Hannover Herausgeber:

Univ.-Prof. Dr.-Ing. Steffen Marx

Leibniz Universität Hannover - Institut für Massivbau

Heft 14, März 2018

Fraunhofer IRB Verlag 


\section{Herausgeber \\ Univ.-Prof. Dr.-Ing. Steffen Marx \\ Leibniz Universität Hannover \\ Institut für Massivbau \\ Appelstraße $9 \mathrm{~A}$ \\ 30167 Hannover}

Alle Rechte vorbehalten

Dieses Werk einschließlich aller seiner Teile ist urheberrechtlich geschützt. Jede Verwertung in anderen als den gesetzlich zugelassenen Fällen bedarf deshalb der vorherigen schriftlichen Einwilligung des Herausgebers.

(C) 2018 by Univ.-Prof. Steffen Marx

Leibniz Universität Hannover

Institut für Massivbau

ISBN (Print): 978-3-7388-0102-6

ISBN (E-Book): 978-3-7388-0103-3

\section{Fraunhofer IRB Verlag}

Fraunhofer-Informationszentrum Raum und Bau IRB

Postfach 8004 60, 70504 Stuttgart

Telefon $0711970-2500$

Telefax 0711 970-2508

E-Mail irb@irb.fraunhofer.de

URL http://www.baufachinformation.de 


\title{
Simulation und Regelung von \\ Resonanzversuchsständen zur Untersuchung der Bauteilermüdung
}

\author{
Von der Fakultät für Bauingenieurwesen und Geodäsie der \\ Gottfried Wilhelm Leibniz Universität Hannover \\ zur Erlangung des akademischen Grades
}

\author{
Doktor der Ingenieurwissenschaften
}

$$
\text { Dr.-Ing. }
$$

genehmigte Dissertation

von

Herrn Ralf Herrmann, M.Sc.

aus

Karl-Marx-Stadt 
Hauptreferent: $\quad$ Univ.-Prof. Dr.-Ing. Steffen Marx

Korreferent: $\quad$ Univ.-Prof. Dr.-Ing. habil. Peter Mark

Kommissionsmitglied: Univ.-Prof. Dr.-Ing. Nabil A. Fouad

Vorsitz:

Univ.-Prof. Dr.-Ing. Martin Achmus

Tag der Promotion: $\quad$ 23.10.2017 
Für Carmen 


\section{Danksagungen}

Großen Dank möchte ich meinem Doktorvater, Herrn Univ.-Prof. Dr.-Ing. Steffen Marx aussprechen, der es mir ermöglicht hat in einer interdisziplinären Zusammenarbeit den bisher größten Resonanzversuchsstand für Bauteiluntersuchungen mit zu entwickeln. Besonders möchte ich ihm danken für das Vertrauen, die unentwegte Ermutigung bei der Realisierung dieses Vorhabens und für die engagierte Betreuung, die zum Entstehen dieser Arbeit geführt hat.

Ohne meinen Kollegen, Herrn Dipl.-Ing. Sebastian Schneider, wäre diese Arbeit nicht möglich gewesen. Ihm gebührt die Anerkennung für die Planung und Entwicklung des Spannfelds und die mechanische Konstruktion der Versuchsstände. Vielen Dank für die unzähligen Gespräche, die mir die Welt des Bauingenieurwesens erklärt haben und die viele Zeit, die wir in den Aufbau und die Ausstattung der Versuchshalle, der Versuchsstände, die Durchführung und Auswertung von Ermüdungsversuchen investiert haben, von denen nun noch Generationen an Doktoranden profitieren werden. Danken möchte ich auch meinem Kollegen Herrn Dipl.-Ing. Jens Piehler, M.Sc. für die vielen Gespräche in der Promotionszeit über Rückschläge und Erfolge.

Ich danke Herrn Univ.-Prof. Dr.-Ing. habil. Peter Mark für das entgegengebrachte Interesse an meiner Arbeit und die Übernahme des Korreferats, ebenso wie Herrn Univ.-Prof. Dr.-Ing. Nabil Fouad für die wissenschaftliche Begleitung und die Tätigkeit in der Promotionskommission, sowie Herrn Univ.-Prof. Dr.-Ing. Martin Achmus für die Übernahme des Vorsitzes.

Mein besonderer Dank geht an meine Mutter, für die Unterstützung und die Ermutigung diese Arbeit zu einem guten Abschluss zu bringen.

Ralf Herrmann

Hannover, 2017. 


\section{Abstract}

The fatigue behavior of many structural components in civil engineering needs to be investigated in detail to proof the safety and reliability. The existing concepts of structural testing machines are not sufficient to fulfill the requirements regarding an economic and reliable experimental procedure for these tests on large structural elements. The focus of this thesis is the development of a concept for an efficient and adoptable design of a resonant testing setup to realize fatigue tests in the very high cycle fatigue range. A resonant testing system applies a harmonic excitation load from an imbalance rotor to a specimen with a low damping factor at its eigenfrequency which results in an amplified stress. To achieve a constant stress level under continuous changing properties of the test object a closed loop control was developed that refers an arbitrary reference for the dynamic load level (e.g. force, strain or mechanical stress amplitude) to a generalized control reference. For the synchronization of the rotation angle of the uncoupled imbalance rotors an angular positioning method based on a state machine was developed, that only depends on simple position sensors. A complete digital model of the resonant testing system that simulates the mechanical, electrical and control behavior can be used to perform the test virtually before the realization of the test stand. The described simulation environment can be used to analyze the feasibility of the planned tests and the quality of the stress generation. Three dynamic effects are examined that results from the interaction between imbalance rotor and the vibration movement of the specimen. The design concept is implemented on three fatigue test stands. A reinforced concrete beam has been tested in the very high cycle range of $1,0 \cdot 10^{8}$ load cycles. The utilisation of the test concept to railroad railway fatigue tests significantly lowered the duration of the test by the factor 4 and the energy consumption by the factor 792 in comparison with hydraulic testing systems. The requirements for continuous vibration tests are reached for both simulations and realized test stands.

Keywords:

fatigue, resonance, control, structural testing, simulation, digital twin 


\section{Zusammenfassung}

Für viele Bauteilkonstruktionen im Bauingenieurwesen sind Untersuchungen des Ermüdungsverhaltens essentiell zum Nachweis von Sicherheit und Zuverlässigkeit erforderlich. Die dafür existierenden Konzepte für dynamische Prüfmaschinen reichen jedoch nicht aus, um den Anforderungen an eine wirtschaftliche und gleichzeitig qualitativ hochwertige Versuchsdurchführung an großen Bauteilen zu entsprechen. Im Mittelpunkt dieser Arbeit steht die Entwicklung eines Konzepts zur effizienten und individuell an jede Versuchskonfiguration anpassbare Auslegung von Resonanzversuchsständen, um Ermüdungsuntersuchungen an großen Bauteilen im Langzeitfestigkeitsbereich zu realisieren. Bei einem Resonanzversuchsstand wird der schwingungsfähige Probekörper durch eine harmonische Kraftanregung mittels Unwuchtantrieben nahe der Eigenfrequenz belastet und aufgrund der schwachen Dämpfung des Schwingungssystems eine Vervielfachung der Beanspruchung des Bauteils erzeugt. Um trotz der sich bei Ermüdung stetig ändernden Bauteileigenschaften konstante Lastschwingbreiten zu erzeugen, wurde ein Regelungsansatz entwickelt, bei dem die beliebig gewählte Führungsgröße (z. B. Kraft-, Dehnungs- oder Spannungsschwingbreite) auf die dynamisch erzeugte Kraft als generalisierte Regelgröße überführt wird. Zur elektrischen Synchronisation von Unwuchtantrieben wurde ein auf der Theorie der Zustandsautomaten basierendes Bestimmungsverfahren der Winkeldifferenz zwischen zwei Antrieben entwickelt, dass mit einfachen Positionssensoren realisierbar ist.

Durch die entwickelte Simulationsumgebung kann bei jeder Versuchsauslegung ein komplettes digitales Modell des Resonanzversuchsstands erstellt werden, dass das elektrische, regelungstechnische und mechanische Verhalten des geplanten Experiments virtuell vor der Umsetzung simuliert und so die Realisierbarkeit mit den gewählten Antriebskomponenten und die Qualität der Beanspruchungserzeugung geprüft werden. Es wurden drei dynamische Effekte mit dieser Simulation untersucht, die sich aus der Interaktion zwischen dem schwingenden Bauteil und den Unwuchtantrieben ergeben.

Das Resonanzversuchsstandskonzept wurde erfolgreich an drei Resonanzversuchsständen realisiert. Es wurde ein Stahlbetonbalken im Langzeitfestigkeitsbereich mit 1, $0 \cdot 10^{8}$ Lastwechseln untersucht und nachgewiesen, dass auch bei niedrigen Spannungsschwingbreiten Ermüdungsschädigung auftritt. Durch die Anwendung dieser Prüftechnik auf die Untersuchung der Gestaltsfestigkeit von Eisenbahnschienen wurde die Prüfdauer um den Faktor 4 verkürzt und der Energiebedarf um den Faktor 792 im Vergleich zu konventioneller hydraulischer Prüftechnik reduziert. Sowohl in der Simulation, als auch am realen Versuchsstand konnte die Einhaltung der normativen Anforderungen an einen Schwingfestigkeitsversuch belegt werden.

Schlüsselworte:

Ermüdung, Resonanz, Regelung, Bauteiluntersuchung, Simulation, digitaler Zwilling 


\section{Inhaltsverzeichnis}

1 Einleitung 1

1.1 Motivation und Hintergrund . . . . . . . . . . . . . . . . . 1

1.2 Zielsetzung und Aufbau der Arbeit . . . . . . . . . . . . . . . 4

2 Stand der Technik $\quad 7$

2.1 Notwendigkeit von Bauteilprüfungen . . . . . . . . . . . . 7

2.2 Resonanzbasierte Prüfstände zur Bauteilprüfung . . . . . . . . . . . . . 9

2.2.1 Historische Prüfmaschinen . . . . . . . . . . . . . . . . . . . . 9

2.2.2 Industrielle Resonanzprüfmaschinen . . . . . . . . . . . . . . . 10

2.2.3 Resonanzprüfmaschine der TU Wien . . . . . . . . . . . . . . 11

2.2.4 Resonanzprüfmaschine für Rohrverbindungen . . . . . . . . . . . . 14

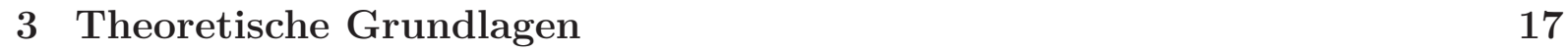

3.1 Grundlagen der mathematischen Modellierung von Schwingungssystemen . 17

3.2 Berechnung des Ersatzsystems des Biegebalkens . . . . . . . . . . . . 21

3.3 Krafterzeugung von Unwuchtantrieben . . . . . . . . . . . . . . . 23

3.4 Erzeugung einer gerichteten Unwuchtkraft . . . . . . . . . . . . 27

3.5 Interaktion zwischen Schwingungssystem und Unwuchtantrieb . . . . . . . 30

3.6 Auslegung der Unwuchtmassen . . . . . . . . . . . . . . . . . . 32

3.7 Berücksichtigung der Federmassen . . . . . . . . . . . . . . . . . . . . 34

3.8 Antriebsmaschinen . . . . . . . . . . . . . . . . . . . 36

3.9 Modellierung einer Asynchronmaschine . . . . . . . . . . . . . . . . . . . . 40

3.10 Grundlagen von Steuerung und Regelung . . . . . . . . . . . . . . . . . . . 44

3.11 Grundlagen der dynamischen Simulation . . . . . . . . . . . . . 46

3.11.1 Standardverfahren der dynamischen Simulation . . . . . . . . . . 46

3.11 .2 Modellbeschreibung für dynamische Simulationen . . . . . . . . . . 48

3.11.3 Numerische Lösungsverfahren für gewöhnliche Differentialgleichungen 48

3.11 .4 Anwendungen der dynamischen Simulation . . . . . . . . . . . . . . 49 
4.1 Zielstellungen bei der Anwendung von Resonanzversuchsständen für Großversuche . . . . . . . . . . . . . . . . . . . . . 53

4.2 Mechanisches Versuchsprinzip . . . . . . . . . . . . . . . 54

4.3 Konzeptioneller Aufbau . . . . . . . . . . . . . . . . . . . . . . 54

4.4 Vorgehen zur Durchführung von Bauteilversuchen . . . . . . . . . . . . 56

4.5 Spannfeld für Resonanzversuchsstände . . . . . . . . . . . . . . . 56

4.6 Systemstruktur eines Resonanzversuchsstands . . . . . . . . . . . . . . 58

5 Steuerung und Regelung des Resonanzversuchsstands $\quad 61$

5.1 Auswahl der Führungsgrößen . . . . . . . . . . . . . . . . . . . . . 61

5.1 .1 Schwingweg des Bauteils . . . . . . . . . . . . . 62

5.1 .2 Schwinggeschwindigkeit des Bauteils . . . . . . . . . . . . 62

5.1 .3 Schwingbeschleunigung des Bauteils . . . . . . . . . . . 63

5.1 .4 Anregende Unwuchtkraft . . . . . . . . . . . . . . . . . . 63

5.1 .5 Auflagerreaktion .................... 64

5.1 .6 Materialdehnung . . . . . . . . . . . . . . . . . . . 64

5.2 Systemtheoretische Struktur der Regelstrecke . . . . . . . . . . . . . . . . 64

5.3 Reglerentwurf . . . . . . . . . . . . . . . . . 66

5.3 .1 Anforderungen an die Regelung . . . . . . . . . . . . . . 66

5.3.2 Regelung der Phasensynchronisation . . . . . . . . . . . . . . . 67

5.4 Regelung der dynamischen Kraft . . . . . . . . . . . . . . . . 73

6 Simulationsmodelle für Resonanzversuchsstände $\quad 77$

6.1 Entwicklung einer Simulationsumgebung: der digitale Zwilling . . . . . . . 77

6.2 Frequenzumrichter . . . . . . . . . . . . . . . . . . 81

6.3 Antriebsmaschine . . . . . . . . . . . . . . . . . 85

6.4 Unwuchtantrieb . . . . . . . . . . . . . . . . . 86

6.5 Schwingungssystem . . . . . . . . . . . . . . . . . 87

6.6 Messsystem . . . . . . . . . . . . . . . . . . . . . 88

6.7 Regelung . . . . . . . . . . . . . . . . . . . . 88

6.8 Simulation von Resonanzversuchen . . . . . . . . . . . . . . . . . . 88

6.8.1 Sommerfeldeffekt . . . . . . . . . . . . . . . . . . . . 89

6.8.2 Schwebungen in der Unwuchtkraft . . . . . . . . . . . . . . . . 90

6.8.3 Selbstsynchronisation der Unwuchtantriebe . . . . . . . . . . . . . . 91

6.8.4 Einschwingverhalten des Einmassenschwingers . . . . . . . . . . . 93

7 Realisierung von Resonanzversuchsständen $\quad 97$

7.1 Versuchsstand 1 - Ermüdungsfestigkeit von Eisenbahnschienen . . . . . . . 97 
7.1.1 Prüfaufgabe . . . . . . . . . . . . . . . . . . . 98

7.1.2 Auslegung des Versuchsstands . . . . . . . . . . . . . . . . . . . 99

7.1.3 Versuchsstandsrealisierung und Ergebnisse . . . . . . . . . . . . . 101

7.1.4 Vergleich zwischen realem Versuchsstand und Simulation . . . . . . 105

7.1 .5 Leistungsaufnahme . . . . . . . . . . . . . . . . . . . 107

7.2 Versuchsstand 2 - Langzeitfestigkeit von Stahlbetonbalken . . . . . . . . . 109

7.2.1 Prüfaufgabe . . . . . . . . . . . . . . . . . . . 109

7.2.2 Auslegung des Versuchsstands . . . . . . . . . . . . . . . . . . 109

7.2.3 Versuchsstandrealisierung und Ergebnisse . . . . . . . . . . . . . . 111

7.3 Versuchsstand 3 - Spannungsumlagerungen in

Spannbeton . . . . . . . . . . . . . . . . . 117

7.3.1 Prüfaufgabe . . . . . . . . . . . . . . . . 117

7.3.2 Auslegung des Versuchsstands . . . . . . . . . . . . . . . . 118

7.3.3 Versuchsstandsrealisierung und Ergebnisse . . . . . . . . . . . . 121

8 Schlussfolgerungen und Ausblick $\quad 125$

8.1 Zusammenfassung . . . . . . . . . . . . . . . . . . . 125

8.2 Der digitale Zwilling im Prüfbetrieb . . . . . . . . . . . . . . 127

8.3 Der digitale Zwilling in der Wartung . . . . . . . . . . . . . . 127

A Tätigkeitsschritte zur Auslegung eines Resonanzversuchsstands $\quad 129$

B Koeffizienten des s-stufigen Runge-Kutta-Verfahrens 131 


\section{Abbildungsverzeichnis}

2.1 Definition der Begriffe Lastamplitude, Lastschwingbreite, Ober-, Mittelund Unterlast . . . . . . . . . . . . . . . . . . . . . . . . . . . . . 8

2.2 Erste Prüfmaschine von J.H SMith, die die Prüfkraft durch rotierende Unwuchtmassen erzeugte, aus $[\mathrm{Sie} 40]$. . . . . . . . . . . . . . . . . 10

2.3 Schnitt und Ansicht der von C. ScHECK gebauten Fliehkraftmaschine, aus

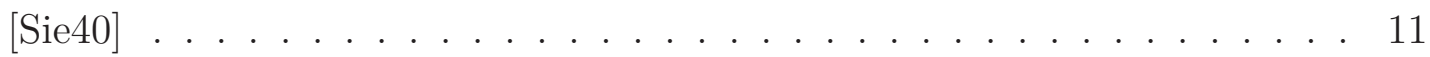

2.4 Ansicht des Schwingungsantriebs der Druck-Zug-Fliehkraftmaschine von C. SchenCK, aus [Leh30] . . . . . . . . . . . . . . . . . . . . . . . . 12

2.5 Industrielle Resonanzprüfmaschine . . . . . . . . . . . . . . . . . . . . . 12

2.6 Resonanzprüfstand für Spannkabel der TU Wien . . . . . . . . . . . . . . 13

2.7 Resonanzprüfmaschine für Bohrgestänge zur Förderung von Öl und Gas der Universität Gent, aus [VW11] . . . . . . . . . . . . . . . . 15

3.1 Einfaches Feder-Masse-Dämpfer-System und zugehöriges Freikörperbild . . 18

3.2 Unwuchtantrieb mit rotierender Punktmasse . . . . . . . . . . . . . . . . . 24

3.3 Amplituden- und Phasenverlauf bei Unwuchtanregung . . . . . . . . . . 26

3.4 Zwei biegesteif gekoppelte Unwuchtantriebe erzeugen Kräfte und Momente in Abhängigkeit von der Drehrichtung und dem Winkelversatz . . . . . . . 27

3.5 Gegenseitige Aufhebung der horizontalen Kraftkomponenten bei versatzfreier Rotation mit $\Delta \varphi=0^{\circ} \ldots \ldots \ldots \ldots$. . . . . . . . . . . 28

3.6 Fliehkraftvektoren und Phasenversatz von zwei Unwuchtantrieben . . . . . 29

3.7 Darstellung der an der Antriebswelle angreifenden Kräfte und Momente . . 31

3.8 Konturenformen von Unwuchtmassen bei Verwendung als Schwingungsantrieb, nach $[\mathrm{DF} 14] \ldots \ldots \ldots$

3.9 Klassifikation von elektrischen Antrieben . . . . . . . . . . . . . . 36

3.10 Schnitt einer Asynchronmaschine mit einer Polpaarzahl $p=1$ und einem dreisträngigen Netz . . . . . . . . . . . . . . . . . . . . . . . 38

3.11 Die Drehzahl-Drehmoment-Kennlinie einer Asynchronmaschine bei Betrieb am starren Netz . . . . . . . . . . . . . . . . . . . . . . . 39

3.12 Ersatzschaltbild einer Asynchronmaschine anhand der Grundwellenbetrachtung eines Strangs . . . . . . . . . . . . . . . . . . . . . 41 
3.13 Ersatzschaltbild einer Asynchronmaschine im $q$, d-Koordinatensystem . . . 42

3.14 Gegenüberstellung der prinzipiellen Blockschaltbilder von Steuerung und Regelung . . . . . . . . . . . . . . . . . . . . 45

3.15 Darstellung eines linearen gewöhnlichen Differentialgleichungssystems als Blockschaltbild, wie es in regelungstechnischen Simulationen eingegeben wird

4.1 Mechanisches Prinzip eines Resonanzversuchsstands für Bauteile mit großen Abmessungen, konzipiert als Einmassenschwinger . . . . . . . . . . . . . 54

4.2 Realisierungskonzept für Resonanzprüfstände als Spannfeldaufbauten . . . 55

4.3 Spannfeld zur Realisierung von unterschiedlichen Resonanzversuchsständen entsprechend der Erfordernisse des Bauteilversuchs . . . . . . . . . . . . 57

4.4 Probekörperkonfigurationen auf dem Spannfeld unter Nutzung von ein, zwei oder vier Federkellern . . . . . . . . . . . . . . . . . . . . . . 58

4.5 Systemstruktur, die bei jeder aufgabenspezifischen Realisierung eines Resonanzversuchsstands anzustreben ist (hier für einen Versuchsstand mit vier Unwuchtantrieben)

5.1 Systemtheoretische Struktur der Resonanzprüfmaschine zur Bauteiluntersuchung bei Einsatz von zwei Unwuchtantrieben . . . . . . . . . . . . . . . 65

5.2 Systemtheoretische Struktur der Resonanzprüfmaschine zur Bauteiluntersuchung bei Einsatz von vier Unwuchtantrieben . . . . . . . . . . . . . 66

5.3 Industrieller Drehgeber für einen Unwuchtantrieb, nach [Net08] . . . . . . 68

5.4 Robuste Rotorpositionsbestimmung von Unwuchtantrieben durch Hall-Sensoren bei deren Anwendung in Resonanzversuchsständen . . . . . . . . . . . . . . 69

5.5 Zustandsautomat zur Realisierung einer robusten Bestimmung der Phasendifferenz von zwei Unwuchtantrieben . . . . . . . . . . . . . . . . . . . 71

5.6 Schaltverhalten der zwei Positionsgeber von elektrisch synchronisierten Unwuchtantrieben. Fallende Flanken stellen das Eintreten der Magneten in die Erfassungsbereiche der beiden Sensoren dar. . . . . . . . . . . . . . . . . . 72

5.7 Regelkreis zur Führungsgrößenregelung mit Linearisierung des Eingangs/Ausgangsverhaltens . . . . . . . . . . . . . . . . . 75

6.1 Kopplung von FE-Simulation, MK-Simulation und Regelungssimulation . . 78

6.2 Realisierungskonzept für Resonanzversuchsstände für großmaßstäbliche Bauteiluntersuchungen . . . . . . . . . . . . . . . . . . . . 79

6.3 Bestandteile der Gesamtsimulation mit Beispielen für unterschiedliche Teilkomponenten ......................... . . 81 
6.4 Elektrischer Aufbau eines IGBT-Frequenzumrichters mit drei Strängen, die über sechs IGBT geschaltet werden . . . . . . . . . . . . . . . . 82

6.5 Modulierte Signale der Sinusoidal Pulse Width Modulation (SPWM) zur Generierung der Schaltsignale und der Strangspannungen durch das Teilmodell Frequenzumrichter . . . . . . . . . . . . . . . . . . . . . . . . 84

6.6 Hochlauf eines idealen Antriebs mit konstantem elektromagnetischen Moment $T_{E}=1,25 \mathrm{Nm}$ durch die Resonanzfrequenz des Probekörpers bei $\omega_{0}=125 \mathrm{rad} / \mathrm{s}$

6.7 Unterschiede in den Lagerreibungen und den Drehzahlen von nicht elektrisch synchronisierten Unwuchtantrieben führen in der Simulation zu einer überlagerten Schwingung der Auslenkungsamplitude, hervorgerufen durch eine Schwebung in der Unwuchtkraft. . . . . . . . . . . . . . . . . . . . . . 92

6.8 Anlaufen des Unwuchtantriebs ohne elektrische Synchronisation bei einem Bauteil mit großer Ersatzmasse. Es tritt keine Selbstsynchronisation auf. . 93

6.9 Anlaufen des Unwuchtantriebs ohne elektrische Synchronisation bei einem Bauteil mit kleiner Ersatzmasse. Es tritt eine Selbstsynchronisation der Unwuchtantriebe auf. Der Winkelversatz nähert sich einem stationären Endwert von $\Delta \varphi=0^{\circ}$ an. . . . . . . . . . . . . . . . . . . . . . . . . 94

6.10 Einschwingvorgang eines Einmassenschwingers bei unterschiedlichen Anregungsfrequenzen . . . . . . . . . . . . . . . . . . 95

7.1 Vierpunktbiegeversuch zur Untersuchung der Ermüdungsfestigkeit von Ei-

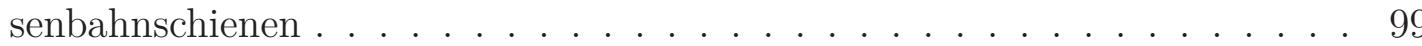

7.2 Durch experimentelle Modalanalyse ermittelte Schwingungseigenform der ersten Biegeeigenschwingung des Probekörpers bei einer Frequenz von 20,6 Hz102

7.3 Realisierter Versuchsstand zur Untersuchung des Ermüdungsverhaltens von Eisenbahnschienen . . . . . . . . . . . . . . . . . 103

7.4 Bruchfläche einer Schiene nach 1271709 Lastwechseln, die bei einer Spannungsschwingbreite am Schienenfuß von $290 \mathrm{MN} / \mathrm{m}^{2}$ geprüft wurde . . . . 103

7.5 Verlauf der mechanischen Spannung am Schienenfuß und der Durchbiegung in Feldmitte während des Ermüdungsversuchs (oben), sowie der mechanischen Spannungsschwingbreite als Regelgröße (unten) . . . . . . . . . . . . 104

7.6 Vergleich zwischen realem Ermüdungsversuch von Schienen am Versuchsstand MW03 (links) und dessen Simulation (rechts) . . . . . . . . . . 106

7.7 Schaltplan der maschinenseitigen Leistungsmessung an einem Unwuchtantrieb während des Versuchs . . . . . . . . . . . . . . . . . . . . . . 108

7.8 Leistungsaufnahmen für einen Unwuchtantrieb für unterschiedliche Spannungsschwingbreiten am Schienenfuß . . . . . . . . . . . . . . . 108 
7.9 Bewehrungszeichnung und Messstellenplan für Probekörper zur Durchführung eines Very-High-Cycle-Fatigue-Tests . . . . . . . . . . . . . . . . . . . 110

7.10 Lasteinleitungskonstruktion zur Untersuchung des Ermüdungsverhaltens von Stahlbetonbalken . . . . . . . . . . . . . . . . . . . . . . 112

7.11 Realisierter Versuchsstand zur Untersuchung des Ermüdungsverhaltens von Stahlbetonbalken im Very-High-Cycle-Fatigue-Bereich (nach Versuchsende) 113

7.12 Gemessenes Schwingungsverhalten des Stahlbetonbalkens beim Übergang vom ungerissenen in den gerissenen Zustand im Resonanzversuch . . . . . . 114

7.13 Verlauf von maximaler und minimaler Durchbiegung des Probekörpers in Feldmitte im Langzeitversuch Stahlbetonermüdung . . . . . . . . . . . . 116

7.14 Verlauf von Oberspannung und Unterspannung im Langzeitversuch Stahlbetonermüdung . . . . . . . . . . . . . . . . . . . . 116

7.15 Gerissene Bewehrung des Stahlbetonbalkens . . . . . . . . . . . . . 117

7.16 Rissbild des Stahlbetonbalkens . . . . . . . . . . . . . . . . . . 117

7.17 Probekörper aus Spannbeton . . . . . . . . . . . . . . . . . . . 118

7.18 Lasteinleitung für Spannbetonbalken mit vier Unwuchtantrieben und Schienenabschnitten zur Messung der Auflagerkräfte . . . . . . . . . . . . . . . 121

7.19 Realisierter Versuchsstand zur Untersuchung der Spannungsumlagerung in Spannbeton . . . . . . . . . . . . . . . . . . . . 122

7.20 Schwingungseigenform des Probekörpers bei einer Frequenz von $f_{01}=15,7 \mathrm{~Hz} 123$

7.21 Schwingungseigenform des Probekörpers bei einer Frequenz von $f_{02}=18,2 \mathrm{~Hz} 123$

7.22 Schwingungseigenform des Probekörpers bei einer Frequenz von $f_{03}=49,8$ Hz123

7.23 Verlauf der gemessenen Auflagerkraft und Durchbiegung in Feldmitte bei geregelter Auflagerkraftschwingbreite in Laststufen von $100 \mathrm{kN}$ bis zu einer Kraftschwingbreite von $600 \mathrm{kN}$, danach $650 \mathrm{kN}, 700 \mathrm{kN}$ und $725 \mathrm{kN}$ Kraftschwingbreite . . . . . . . . . . . . . . . . . . . . . . . 124 


\section{Tabellenverzeichnis}

3.1 Frequenzgleichungen und Eigenschwingungsbiegelinien für die vier StandardEuler-Stäbe nach $[$ Pet00]. . . . . . . . . . . . . . . . . . . . . 22

3.2 Eigenfrequenzen für Balken nach $[$ Pet00] . . . . . . . . . . . . . . . . 22

3.3 Anzahl der verfügbaren Typen von Unwuchtantrieben von verschiedenen Herstellern, klassifiziert nach Art der Maschine. Stand: Mai 2015 . . . . . . 37

3.4 Mechanische Synchrondrehzahlen von Drehfeldmaschinen bei einer Netzfrequenz von $50 \mathrm{~Hz} \ldots \ldots \ldots$. . . . . . . . . . . . . . . . 40

5.1 Bedeutung der Ausgabeaktionen des Zustandsautomaten zur Phasensynchronisation . . . . . . . . . . . . . . . . . . . 70

6.1 Modell Frequenzumrichter - feldorientierte Regelung . . . . . . . . . . . . . 83

6.2 Modell Frequenzumrichter - Leistungselektronik . . . . . . . . . . . . . . . 84

6.3 Modell Antriebsmaschine . . . . . . . . . . . . . . . . . . . . . . . . 85

6.4 Modell Unwuchtantrieb . . . . . . . . . . . . . . . . . . . . . . 86

6.5 Modell Schwingungssystem . . . . . . . . . . . . . . . . 87

6.6 Modell Messsystem . . . . . . . . . . . . . . . . . . 88

6.7 Modell Regelung . . . . . . . . . . . . . . . . . . . . . . . . 89

7.1 Zusammensetzung der schwingenden Gesamtmasse für den Versuchsstand Schienenermüdung . . . . . . . . . . . . . . . . . . . . 100

7.2 Kenndaten Maschinentyp Netter NEG 25420 . . . . . . . . . . . . . . . 101

7.3 Zusammensetzung der schwingenden Gesamtmasse für den Versuchsstand Stahlbetonermüdung . . . . . . . . . . . . . . . . . . . 111

7.4 Kenndaten Maschinentyp Netter NEG 502270 . . . . . . . . . . . . . . . . 112

7.5 Kenndaten Maschinentyp Netter NEG 254310 . . . . . . . . . . . . . . . 119

7.6 Zusammensetzung der schwingenden Gesamtmasse für den Versuchsstand ProBeton . . . . . . . . . . . . . . . . . 120 


\section{Abkürzungsverzeichnis}

$\begin{array}{ll}\text { ASM } & \text { Asynchronmaschine } \\ \text { CPU } & \text { Central Processing Unit } \\ \text { DMS } & \text { Dehnungsmessstreifen } \\ \text { EMS } & \text { Einmassenschwinger } \\ \text { FEM } & \text { Finite-Elemente-Methode } \\ \text { FOC } & \text { feldorientierte Regelung } \\ \text { FPGA } & \text { Field Programmable Gate Array } \\ \text { IGBT } & \text { Bipolartransistor mit isolierter Gate- } \\ & \text { Elektrode } \\ \text { MKS } & \text { Mehrkörpersimulation } \\ \text { MOSFET } & \text { Metall-Oxid-Halbleiter-Feldeffekttransistor } \\ \text { PWM } & \text { Pulse Width Modulation } \\ \text { SM } & \text { Synchronmaschine } \\ \text { SPWM } & \text { Sinusoidal Pulse Width Modulation }\end{array}$




\section{Symbolverzeichnis}

$\alpha \quad$ geometrischer Öffnungswinkel einer Unwuchtgeometrie

d Dämpfungskoeffizient in $\frac{N s}{m}$

$d_{0} \quad$ Breite einer Unwuchtscheibe

D Lehrsches Dämpfungsmaß

$e \quad$ Abstand zwischen Massenmittelpunkt und Drehachse

E $\quad$ E-Modul eines Materials

$\vec{e} \quad$ Abstandsvektor zwischen Drehachse und Schwerpunkt der Unwuchtmasse

$E_{\text {pot }} \quad$ potentielle Energie eines Systems

$E_{\text {kin }} \quad$ kinetische Energie eines Systems

$f_{0} \quad$ Eigenfrequenz eines Probekörpers $f_{0}=\frac{\omega_{0}}{2 \pi}$

$F_{z f} \quad$ Betrag der Zentrifugalkraft bei Unwuchtantrieben

$F_{u} \quad$ erzeugte resultierende Kraft mehrerer Unwuchtantriebe

$f_{1} \quad$ elektrische Frequenz des Drehstromnetzes

$F_{d y n} \quad$ Betrag der dynamsch erzeugten Kraft

$\Delta F \quad$ dynamisch erzeugte Kraftschwingbreite aller Unwuchtantriebe (Peak)

$g \quad$ Erdbeschleunigung

$\eta \quad$ Verhältnis zw. Erregerfrequenz und erster ungedämpfter Eigenfrequenz

$h \quad$ Schrittweite bei numerischer Lösung von GDGL

I Flächenträgheitsmoment eines Bauteils

$i_{q} \quad$ momenterzeugender Strom in der q-Achse

$i_{d} \quad$ flusserzeugender Strom in der d-Achse

$J \quad$ Massenträgheitsmoment

$J_{R} \quad$ Trägheitsmoment des Rotors und der Welle der Antriebsmaschine

$J_{u}^{(S)} \quad$ Trägheitsmoment der Unwuchtmasse bei Rotation um Achse des Massenmittelpunkts 
$J_{u} \quad$ Trägheitsmoment der Unwuchtmasse bei Rotation um die Drehachse mit einer Exzentrizität $e$

$k \quad$ Federsteifigkeit

$k^{*} \quad$ Ersatzsteifigkeit des äquivalenten Einmassenschwingers

$l \quad$ Abstand der Rotorachsen von zwei Unwuchtantrieben

$L_{1 h} \quad$ Hauptinduktivität der Statorspule

$L_{2 h} \quad$ Hauptinduktivität der Rotorspule

$L_{1 \sigma} \quad$ Streuinduktivität der Statorspule

$L_{2 \sigma} \quad$ Streuinduktivität der Rotorspule

$m \quad$ Masse

$m^{*} \quad$ Ersatzmasse des äquivalenten Einmassenschwingers

$\mu \quad$ Masse pro Längeneinheit

$m_{u} \quad$ exzentrische Masse eines einzelnen Unwuchtantriebs auf beiden Wellenenden

$\mu_{l} \quad$ Koeffizient der Lagerreibung

$m_{f} \quad$ Masse des Federpakets aller zusätzlichen Zugfedern

$M_{U} \quad$ Unwuchtmoment

$M_{S} \quad$ harmonisches Schwingmoment

$M_{N} \quad$ Nennmoment

$n_{0} \quad$ Umlaufdrehzahl des Statormagnetfelds

$n_{u} \quad$ Anzahl der Unwuchtantriebe zur Erzeugung der Prüfkraft

$n_{N} \quad$ Nenndrehzahl

$\omega_{0} \quad$ ungedämpfte Eigenkreisfrequenz eines schwingungsfähigen Systems

$\omega_{D} \quad$ Eigenkreisfrequenz eines gedämpften schwingungsfähigen Systems

$\omega_{\text {mech }}$ mechanische Kreisfrequenz bzw. Winkelgeschwindigkeit des rotierenden Rotors

$\vec{\omega} \quad$ Vektor der Winkelgeschwindigkeit eines rotierenden Körpers

$\omega_{R} \quad$ Resonanzkreisfrequenz

$\omega_{1} \quad$ Kreisfrequenz der Netzspannung 


\begin{tabular}{|c|c|}
\hline$\omega$ & elektrische Kreisfrequenz des Rotors \\
\hline$p$ & Anzahl der Statorpole \\
\hline$\varrho$ & Dichte eines Werkstoffes \\
\hline$R$ & Außenradius der Unwuchtscheibe \\
\hline$r$ & Innenradius der Unwuchtscheibe \\
\hline$R_{1}$ & el. Widerstand der Statorwicklung \\
\hline$R_{2}$ & el. Widerstand der Rotorwicklung \\
\hline$s$ & $\begin{array}{l}\text { komplexwertige Laplace-Variable der Übertra- } \\
\text { gungsfunktion }\end{array}$ \\
\hline$m_{1}$ & Strangzahl des Stators \\
\hline$s$ & Schlupf \\
\hline$t$ & Zeit \\
\hline$T_{E}$ & $\begin{array}{l}\text { elektromagnetisch erzeugtes Drehmoment, in- } \\
\text { neres Drehmoment }\end{array}$ \\
\hline$T$ & Zeitkonstante \\
\hline$T_{L}$ & $\begin{array}{l}\text { dem Antriebmoment entgegenwirkendes Last- } \\
\text { moment bzw. Gegenmoment }\end{array}$ \\
\hline$T_{J}$ & $\begin{array}{l}\text { Moment, aufgrund des Trägheitsmoments bei } \\
\text { Rotation um eine bestimmte Achse }\end{array}$ \\
\hline$T_{a}$ & $\begin{array}{l}\text { beschleunigend wirkendes Drehmoment, An- } \\
\text { triebsmoment }\end{array}$ \\
\hline$T_{A}$ & Anlaufmoment \\
\hline$T_{K}$ & Kippmoment der ASM am starren Netz \\
\hline$V$ & $\begin{array}{l}\text { Vergrößerungsfunktion bei dynamischer Anre- } \\
\text { gung }\end{array}$ \\
\hline$\Delta \varphi$ & $\begin{array}{l}\text { Winkeldifferenz zwischen den Rotorlagen } \\
\text { zweier Antriebe }\end{array}$ \\
\hline$x(t)$ & zeitabhängige Auslenkung \\
\hline$\ddot{x}(t)$ & zeitabhängige Beschleunigung \\
\hline$\dot{x}(t)$ & zeitabhängige Geschwindigkeit \\
\hline$\ddot{\varphi}(t)$ & zeitabhängige Winkelbeschleunigung \\
\hline$\varphi(\mathrm{t})$ & zeitabhängiger Drehwinkel \\
\hline$\dot{\varphi}(t)$ & zeitabhängige Winkelgeschwindigkeit \\
\hline$\gamma_{u}$ & Verdrehwinkel zwischen den Unwuchtscheiben \\
\hline $\overrightarrow{\boldsymbol{x}}$ & Vektor der Systemzustände \\
\hline$\vec{u}$ & Vektor der Systemeingänge \\
\hline$\vec{y}$ & Vektor der Systemausgänge \\
\hline$\dot{\overrightarrow{\boldsymbol{x}}}$ & Ableitung des Vektors der Systemzustände \\
\hline
\end{tabular}

